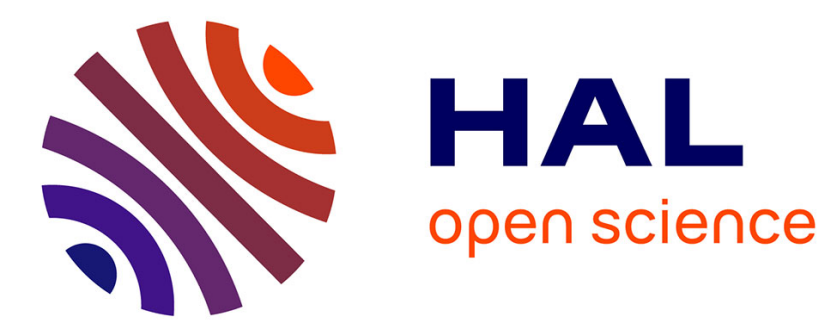

\title{
Les droits et libertés à l'épreuve de la pandémie de Covid-19 en Italie
}

\author{
Jean Fougerouse
}

\section{To cite this version:}

Jean Fougerouse. Les droits et libertés à l'épreuve de la pandémie de Covid-19 en Italie. Cahiers de la recherche sur les droits fondamentaux , 2021. hal-03424480

\section{HAL Id: hal-03424480 \\ https://hal.science/hal-03424480}

Submitted on 18 Nov 2021

HAL is a multi-disciplinary open access archive for the deposit and dissemination of scientific research documents, whether they are published or not. The documents may come from teaching and research institutions in France or abroad, or from public or private research centers.
L'archive ouverte pluridisciplinaire HAL, est destinée au dépôt et à la diffusion de documents scientifiques de niveau recherche, publiés ou non, émanant des établissements d'enseignement et de recherche français ou étrangers, des laboratoires publics ou privés. 


\title{
Les droits et libertés à l'épreuve de la pandémie de Covid-19 en Italie
}

\author{
Jean FOUGEROUSE \\ Maître de conférences (HDR) à l'université d'Angers \\ Centre Jean Bodin (UPRES EA 4337)
}

I. Les bases fragiles des restrictions aux droits et libertés
A. La captation du pouvoir de restriction par l'exécutif national

B. La suprématie conjoncturelle du droit à la santé

II. Le contrôle limité des restrictions aux droits et libertés
A. Une certaine frilosité du juge
B. Droit à la santé versus droit à l'éducation, une illustration d'un contrôle approximatif

Comme de nombreux pays dépourvus d'un cadre constitutionnel clair relatif à l'urgence, l'Italie a dû improviser et adapter ses institutions et ses règles pour lutter contre la propagation de la Covid-19. Ainsi, le gouvernement italien a mobilisé successivement plusieurs ressources pour faire face à la pandémie. Tout d'abord, il a déclarél'état d'urgence sanitaire le 31 janvier $2020^{1}$ pour six mois et l'a prorogé jusqu'au 30 avril $2021^{2}$. Ensuite, le ministre de la Santé a pris des ordonnances le 21 février $202 \mathrm{O}^{3}$ sur la base de l'article 32 de la loi $n^{\circ} 833$ du 23 décembre 1978 relative au service sanitaire national. Face à l'accélération des événements, le gouvernement a eu alors recours aux décrets-lois de l'article 77 de la Constitution: dans un premier temps, le décret-loi du 23 février $202 \mathrm{O}^{4}$ a prévu différentes restrictions et a pour le reste laissé tout pouvoir aux autorités compétentes de prendre des mesures restrictives supplémentaires (on a évoqué alors un "chèque en blanc $»^{5}$ ). Puis le gouvernement a adopté plusieurs décrets-lois, notamment le $17^{6}$ et le 25 mars $2 \mathrm{O}^{7} \mathrm{O}^{7}$, qui ont davantage encadré le pouvoir des autorités administratives; une partie de la doctrine a cependant continué à dénoncer un abandon du Parlement au profit d'un "présidentialisme extra ordinem ${ }^{8}$. Sur la base de ces décrets-lois, le président du Conseil des ministres a adopté des décrets (decreto del presidente del Consisiglio dei ministri - DPCM) ${ }^{9}$ en s'appuyant sur l'expertise du Comité technique scientifique ${ }^{10}$. En outre, la législation nationale ${ }^{11}$ permet au ministre de la Santé de prendre des ordonnances d'urgence ${ }^{12}$, en matière d'hygiène et de santé

1. Délibération du Conseil des ministres prise sur la base du décret législatif no 1 du 2 janvier 2018 ; F. Cerquozzi, «"Stato d'emergenza" e Costituzione», Ius in itinere, 26 mars 2020, revue en ligne.

2. Suite à plusieurs prorogations (le 31 juillet 2020 , le 5 octobre 2020 et le 13 janvier 2021)

3. Gazzetta ufficiale della Repubblica italiana, $\mathrm{n}^{\circ} 44,22$ février 2020

4. Décret-loi $\mathrm{n}^{\circ} 6$ du 23 février 2020.

5. G. Silvestri, «Covid-19 e Costituzione», Unicost, 4 octobre 2020, revue en ligne. Toutes les traductions de l'italien sont les miennes.

6. Décret-loi ${ }^{\circ} 18$ du 17 mars 2020.

7. Décret-loi ${ }^{\circ} 19$ du 25 mars 2020.

8. L. A. Mazzarolli, " "Riserva di legge" e "principio di legalità" in tempo di emergenza nazionale», Federalismi.it, n 1, 2020, revue en ligne.

9. A. Mitrotti, «Il DPCM come inedito strumento di gestione statale dell'emergenza da covid-19", in "Virus in fabula". Diritti e istituzioni ai tempi del COVID-19, G. P. Dolso, M. D. Ferrara, D. Rossi (dir.), Trieste, Edizioni Università di Trieste, 2020, p. 165-182.

10. Créé par le chef du département de la protection civile (ordonnance $n^{\circ} 630$ du 3 février 2020 ).

11. Loi no 833 du 23 décembre 1978, art. 32 et décret législatif du 31 mars 1998, art. 197; décret-loi no 6 du 23 février 2020 , décret-loi no 19 du 25 mars 2020.

12. S. De Gotzen, «I poteri amministrativi al tempo del coronavirus: ordinanze di necessità ed urgenza ", in "Virus in fabula"..., p. 131-145. 
publique, pour l'ensemble du territoire ou sur plusieurs régions, et aux présidents de région et aux maires d'en faire autant dans leurs territoires respectifs (dans le respect des mesures prises au niveau national). Les maires ${ }^{13}$ et surtout les présidents de région ont fait d'ailleurs preuve d'un «hyperactivisme normatif ${ }^{14}$.

L'ensemble de ces textes, et notamment les DPCM, ont permis d'adopter des mesures pour faire face à la propagation du virus mais ont par là même porté atteinte aux droits et libertés garantis par la Constitution. Sans être exhaustif, on peut en rappeler quelques-uns: liberté personnelle ${ }^{15}$, liberté de circulation ${ }^{16}$, liberté religieuse ${ }^{17}$, droit à la vie privée $^{18}$, liberté et droit de vote ${ }^{19}$, liberté de manifester et de se regrouper ${ }^{20}$, droit à l'éducation ${ }^{21}$. On peut donc parler d'une détérioration globale des droits et libertés ${ }^{22}$. Cette altération est d'autant plus flagrante que la République italienne est fondée sur l'inviolabilité des droits de la personne humaine. Aussi, les mesures adoptées pour gérer la pandémie interrogent les fondements du système juridique italien car elles disposent d'une base juridique fragile (I) et les atteintes aux droits et libertés ne sont d'ailleurs limitées que par un contrôle juridictionnel restreint (II).

\section{Les bases fragiles des restrictions aux droits et libertés}

La gestion de la pandémie a conduit à une concentration du pouvoir de restreindre les droits et libertés dans les mains de l'exécutif national (A) au nom d'une suprématie conjoncturelle du droit à la santé (B).

\section{A. La captation du pouvoir de restriction par l'exécutif national}

Le gouvernement italien s'est appuyé sur l'urgence pour évincer le Parlement et sur l'unité pour marginaliser les pouvoirs territoriaux. En effet, la Constitution italienne ne prévoit pas de dispositions permettant explicitement le recours à un état d'urgence sécuritaire ou sanitaire ${ }^{23}$. Toutefois, l'article 78 précise que «les Chambres décident de l'état de guerre et accordent au gouvernement les pouvoirs nécessaires » : il n'a pas été utilisé en dépit de l'usage d'un vocabulaire guerrier pour faire face au virus ${ }^{24}$. L'article 77 prévoit que «dans des cas extraordinaires de nécessité et d'urgence, le gouvernement adopte, sous sa responsabilité, des mesures provisoires ayant force de loi». Enfin, l'article 120 indique: «Le gouvernement peut se substituer aux organes des régions, des villes métropolitaines, des provinces et des communes [...] en cas de danger grave pour la sûreté (incolumità) publique $»^{25}$. En outre, les pouvoirs d'urgence peuvent s'appuyer sur une interprétation de l'article $2^{26}$, qui suppose un équilibre entre droits et devoirs et justifie une législation d'urgence où les devoirs $s^{\prime}$ imposent temporairement aux droits ${ }^{27}$. On peut arriver à des conclusions identiques en s'appuyant sur la salus populi suprema lex esto ${ }^{28}$ et le primum vivere deinde philosophare ${ }^{29}$.

13. G. Alberico, «Le ordinanze contingibili e urgenti nella gestione dell'emergenza sanitaria : il ruolo dei Sindaci nella disciplina del D.L. $33 / 2020$ », Dirittifondamentali.it, $1^{\text {er }}$ juin 2020, revue en ligne; M. Galliani, «I poteri sindacali durante l'emergenza Covid-19", Il diritto amministrativo, $\mathrm{n}^{\circ} 4,2021$, revue en ligne.

14. D. Trabucco, «I "sovrani" regionali: le ordinanze dei presidenti delle giunte al tempo del covid-19", Dirittifondamentali.it, n 1 , 2021, p. 370 , revue en ligne.

15. Constitution italienne, art. 13.

16. Constitution italienne, art. 16; G. P. Dolso, «Emergenza sanitaria e libertà di cicolazione», in “Virus in fabula"..., p. $263-278$.

17. Constitution italienne, art. 19; F. Alicino, "Costituzione e religione in Italia al tempo della pandemia", Stato, Chiese et pluralismo confessionale, $\mathrm{n}^{\circ} 19,2020$, revue en ligne.

18. Constitution italienne, art. 15; F. Filosa, «Il diritto alla privacy nello stato di emergenza ", Dirittifondamentali.it, $\mathrm{n}^{\circ} 2,2020$, p. 847-869, revue en ligne; C. Bergonzini, «Non solo privacy. Pandemia, contact tracing e diritti fondamentali», Dirittifondamentali.it, n ${ }^{\circ}$ 2, 2020, p. 704-730, revue en ligne.

19. Constitution italienne, art. 48; M. G. Rodomonte, «Il diritto ad essere informati quale profilo fondamentale della tutela del diritto di voto e la controversa questione dell'abbinamento del referendum costituzionale alle elezioni. Alcune riflessioni a partire dall'ordinanza n. 195 del 2020 della Corte costituzionale», Osservatorio Associazione italiana dei costituzionalisti (AIC), nº 6, 2020, p. 524-544, revue en ligne; R. Borrello, «L'incidenza della pandemia di Covid-19 sulle votazioni pubblicistiche: alcune riflessioni di diritto interno e comparato ", Nomos, $\mathrm{n}^{\circ} 2,2020$, revue en ligne.

20. Constitution italienne, art. 17 .

21. Constitution italienne, art. 34 .

22. Le droit au logement semble lui renforcé, parce qu'il est instrumental à la santé publique (E. Ponzo, «Una garanzia rafforzata del diritto all’abitazione in periodo di emergenza sanitaria", Osservatorio $A I C, \mathrm{n}^{\circ} 1,2021, \mathrm{p} .61-86$, revue en ligne).

23. Cette omission a été voulue par les constituants au regard de l'usage de l'article 48 de la Constitution de Weimar (G. Rolla, «Profili costituzionali dell'emergenza », Rivista AIC, $\mathrm{n}^{\circ}$ 2, 2015, revue en ligne).

24. Voir par exemple, «L'Italia dichiara guerra al Coronavirus [L'Italie déclare la guerre au virus], voli bloccati e stato d'emergenza: "Spegnere il focolaio prima del vaccino" ", Il riformista, 31 janvier 2020

25. L'incolumità inclut en effet la santé publique et notamment la lutte contre les épidémies (Enciclopedia Treccani, «Incolumità pubblica», en ligne: https://www.treccani.it/enciclopedia/incolumita-pubblica_\%28Enciclopedia-Italiana\%29).

26. «La République reconnaît et garantit les droits inviolables de l'homme, comme individu et comme membre de formations sociales où s'exerce sa personnalité, et exige l'accomplissement des devoirs de solidarité politique, économique et sociale auxquels il ne peut être dérogé».

27. B. Caravita, "L'Italia ai tempi del coronavirus: rileggendo la Costituzione italiana», Federalismi.it, n 6, 2020, revue en ligne; V. Baldini, «Emergenza costituzionale e Costituzione dell'emergenza. Brevi riflessioni (e parziali) di teoria del diritto", Dirittifondamentali.it, $\mathrm{n}^{\circ}{ }^{1,}, 2020$, p. 893-905, revue en ligne.

28. Cicéron, De legibus, 3.8; D. Mantovani, «Quand la santé devient politique», Fondation Collège de France, mai 2020, en ligne: https://www. fondation-cdf.fr/2020/05/13/quand-la-sante-devient-politique/.

29. M. Luciani, «Il sistema delle fonti del diritto alla prova dell'emergenza», Rivista AIC, 10 avril 2020, p. 113, revue en ligne; «face à une situation d'urgence le Parlement et le gouvernement ont le droit, le pouvoir et [...] le devoir d'adopter une législation d'urgence» selon la Cour constitutionnelle (Corte costituzionale - CC) (CC, $\mathrm{n}^{\circ} 15$ de 1982). 
Quoi qu'il en soit, il ressort des dispositions précédentes qu'il existe implicitement un «statut minimum de l'urgence constitutionnelle en cas de crise sanitaire nationale, qui établit le rôle central de la fonction législative $»^{30}$ en tant que garantie constitutionnelle. L'utilisation des DPCM par le gouvernement pour restreindre les droits et libertés constituerait ainsi un renoncement au principe de légalité, de même qu'en raison des diverses réserves de loi établies par la Constitution, un acte ayant force de loi comme un décret-loi n'étant pas suffisant ${ }^{31}$. Il est vrai qu'en temps normal le juge constitutionnel défend un principe de «légalité substantielle» imposant que les actes pris par l'exécutif soient déterminés dans leurs modalités d'exercice par la loi ${ }^{32}$.

Mais à l'opposé on a pu soutenir que les mesures de contraintes ont été globalement acceptées par la population et par une majorité de la doctrine ${ }^{33}$ au nom de la protection de la santé individuelle et publique, de sorte que les normes produites malgré leur déficience seraient validées par cette adhésion générale ${ }^{34}$. Le Conseil d'État ${ }^{35}$ et la Cour constitutionnelle ${ }^{36}$ semblent se rallier à cette vision. Toutefois, un juge pénal a décidé de ne pas appliquer le DPCM du 8 mars 2020 pour cause d'inconstitutionnalité: «L'obligation de rester chez soi constitue une mesure restrictive de la liberté personnelle ${ }^{37}$ alors que l'article 13 de la Constitution prévoit que les mesures restrictives de cette liberté ne peuvent être adoptées que «par un acte motivé de l'autorité judiciaire et dans les seuls cas et formes prévues par la loi». Le juge en déduit deux corollaires: 1) un DPCM «ne peut pas prévoir une limitation à la liberté personnelle, car il s'agit d'une source purement réglementaire de rang secondaire et non d'un acte normatif ayant force de loi $»^{38} ; 2$ ) la Constitution «ne permet même pas à une loi (ou un acte ayant force de loi comme le décret-loi) de prévoir de manière générale et abstraite une obligation de rester au domicile à l'encontre d'une pluralité indéterminée de citoyens ${ }^{39}$. Dans une autre affaire, le président de la République, au nom du gouvernement, a annulé ${ }^{40}$, sur la base de l'avis préalable du Conseil d'État ${ }^{41}$, l'ordonnance par laquelle le maire de Messine avait imposé que les voyageurs désirant entrer en Sicile par cette ville devaient s'enregistrer préalablement. Dans son avis, le Conseil d'État a précisé que le maire avait posé des obligations de faire incompatibles avec l'article 23 de la Constitution qui impose l'intervention préalable du législateur.

L'intervention de l'exécutif national a aussi marginalisé les autorités territoriales au nom de l'unité nécessaire. Ainsi, la Cour constitutionnelle a annulé la loi régionale de la Vallée d'Aoste qui allégeait les mesures prises au niveau national (en autorisant des activités interdites au niveau national) car

[...] la matière objet de l'intervention législative régionale relève de la compétence exclusive de l'État au titre de la «prophylaxie internationale» (article 117 de la Constitution), laquelle inclut toute mesure permettant de lutter contre une pandémie sanitaire en cours ou pour la prévenir ${ }^{42}$.

La Covid-19 étant notoirement une pandémie, la «prophylaxie internationale concerne les normes qui garantissent "l'uniformité au niveau national de la réalisation des programmes élaborés aux niveaux international et supranational" ${ }^{43}{ }^{44}$. La compétence de l'État est exclusive: "La matière de la prophylaxie internationale a un objet bien précis, qui inclut la prévention et la lutte contre les maladies pandémiques, tel qu'il occupe la totalité de ce domaine ${ }^{45}$ car $«$ des raisons logiques plutôt que juridiques ${ }^{46}$ justifient

[...] la nécessité d'une réglementation unitaire, de nature nationale, permettant de préserver l'égalité des personnes

30. M. Calamo Specchia, "Principio di legalità e stato di necessità al tempo del COVID-19", Osservatorio AIC, $\mathrm{n}^{\circ}$ 3, 2020, p. 152, revue en ligne.

31. A. Della Bella, «L'allontanamento dal domicilio del soggetto positivo al covid tra problemi di diritto transitorio e inesistenza dei provvedimenti di quarantena », Sistema penale, 16 mars 2021, revue en ligne; G. L. Gatta, «I diritti fondamentali alla prova del coronavirus. Perché è necessaria una legge sulla quarantena», Sistema penale, 2 avril 2020 , revue en ligne.

32. CC, $\mathrm{n}^{\circ} 115$ de 2011; mais le juge interprète «la loi» comme tout acte ayant force de loi (ex. les décrets-lois).

33. Pour une opposition au nom de la souveraineté du peuple, voir L. R. Perfetti, «Sullo statuto costituzionale dell'emergenza. Ancora sul diritto pubblico come violenza o come funzione dei diritti della persona ", Persona e Amministrazione, n 2, 2020, p. 78, revue en ligne.

34. Dans ce sens, voir V. Crisafulli, Lezioni di diritto costituzionale, t. II, L'ordinamento costituzionale italiano. Le fonti normative, La Corte costituzionale, Padoue, CEDAM, 1984, p. 165.

35. «Les mesures limitatives au déroulement ordinaire de la vie sociale [...] n’ont pas produit de modifications irréversibles de nos droits constitutionnels [...] et ont été vues par les citoyens comme légitimes, car elles étaient perçues comme fonctionnelles pour la protection de la santé», selon le rapport annuel du 16 février 2021 du Conseil d'État (Consiglio di Stato - CS) (en ligne: https://www.ildirittoamministrativo.it/Relazioneattivit\% $\mathrm{C}_{3} \%$ Ao-della-giustizia-amministrativa-Presidente-Consiglio-Stato/ted736).

36. $\mathrm{CC}, \mathrm{n}^{\circ} 37$ de 2021 .

37. Tribunal de Reggio Emilia, $n^{\circ}$ 54, 27 janvier 2021. Pour ce juge, l'interdiction de se rendre en tout lieu hors de son domicile est une atteinte à la liberté personnelle (Constitution italienne, art. 13; CC, nº 68 de 1964); A. Buzzoni, «DPCM illegittimi per lesione della libertà personale e non punibilità », Diritto \& Diritti, 15 mars 2021, revue en ligne.

38. Tribunal de Reggio Emilia, $n^{\circ} 54,27$ janvier 2021.

39. Ibid.

40. Décret du président de la République du 9 avril 2020 sur la base de l'article 138 du décret législatif $n^{\circ} 267$ de 2000 .

41. CS, avis $\mathrm{n}^{\circ} 735$ de 2020 .

42. $\mathrm{CC}, \mathrm{n}^{\circ} 37$ de 2021

43. CC, $\mathrm{n}^{\circ} 5$ de 2018; V. Baldini, «Emergenza sanitaria e personalismo "asimmetrico" nelle politiche regionali», Dirittifondamentali.it, $\mathrm{n}^{\circ}$ 1, 2020, p. 1341-1353, revue en ligne.

44. CC, $\mathrm{n}^{\circ} 37$ de 2021

45. Ibid.

46. CC, $\mathrm{n}^{\circ} 5$ de 2018 . 
dans l'exercice du droit fondamental à la santé et en même temps de protéger l'intérêt de la communauté ${ }^{47}$.

De même, tous les aspects de la lutte contre la pandémie (restrictions, quarantaine, mesures thérapeutiques, vaccination, collecte des données...) relèvent de la compétence de l'État: "[...] les choix adoptés au titre de la prophylaxie internationale [...] ne peuvent prétendre à la rationalité que s'ils obéissent à une direction unitaire et à une vision d'ensemble ${ }^{48}$. Il n'y a donc pas de place pour une "politique régionale autonome de la pandémie ${ }^{49}$, seule restant possible une adaptation régionale «par l'adoption d'actes administratifs, en raison de leur flexibilité $»{ }^{50}$.

En effet, les exécutifs régionaux et locaux ont paradoxalement conservé une certaine capacité d'action pour adopter des restrictions aux droits et libertés alors même

[...] qu'une gestion unitaire de la crise est nécessaire pour éviter que les interventions régionales et locales compromettent la stratégie globale de la gestion de la pandémie, surtout $[\ldots]$ s'il s'agit de limiter les libertés constitutionnelles ${ }^{51}$.

Il est vrai que ces acteurs peuvent prendre des ordonnances urgentes dans le cadre de leurs territoires respectifs $^{52}$. Ces mêmes ordonnances peuvent être prises sur la base de l'article 3 du décret-loi no 19 du 25 mars 2020, entre deux $\mathrm{DPCM}^{53}$, sachant que leurs effets s'éteignent avec le DPCM suivant leur adoption, qu'elles doivent être plus restrictives que les mesures prévues par le décret-loi lui-même, qu'elles doivent être justifiées par l'aggravation du risque sur ce territoire et que les exécutifs régionaux et locaux doivent intervenir «de manière subsidiaire et dans le respect des principes d'adaptation et de proportionnalité ${ }^{54}$. On peut donc dire qu'il existe « une place pour des règles complémentaires au niveau régional en raison de la diversité des situations de la propagation du virus d'une région à l'autre ${ }^{55}$. Mais la prééminence de l'exécutif national sur le législateur et les autorités territoriales s'inscrit dans une situation exceptionnelle:

[...] pour la première fois depuis la guerre, des dispositions fortement restrictives des droits fondamentaux [...] ont été adoptées et appliquées au nom d'une valeur encore plus importante, générale et de rang constitutionnel, la santé publique, c'est-à-dire la santé de tous les citoyens ${ }^{56}$.

C'est à ce titre que l'on peut parler d'une suprématie conjoncturelle du droit à la santé.

\section{B. La suprématie conjoncturelle du droit à la santé}

La place des droits fondamentaux dans la Constitution italienne est déterminante et fondatrice du système dans son ensemble. La Cour constitutionnelle leur attribue d'ailleurs une valeur particulière, par le biais des principes suprêmes de l'ordonnancement juridique qui empêchent toute révision constitutionnelle les altérant et forment une limite à la primauté du droit européen ${ }^{57}$. Le droit à la santé est établi par l'article 32 de la Constitution: «La République protège la santé en tant que droit fondamental de l'individu et intérêt de la collectivité ${ }{ }^{8}$. Or, les restrictions aux autres droits et libertés reposent sur la supériorité du droit de la santé sur ceux-ci. Toutefois, la Cour constitutionnelle estime que les droits constitutionnellement protégés sont d'égale valeur juridique, qu'il n'y a pas de droit absolu ${ }^{59}$ qui s'imposerait aux autres ${ }^{60}$. Dès lors qu'aucun droit ne s'impose de manière permanente et que plusieurs droits viennent à s'appliquer à une même situation, c'est au législateur de concilier ces droits. Cette conciliation est de nature politique et suppose l'utilisation d'une marge d'appréciation suffisante ${ }^{61}$. Ainsi, il n'y a pas de hiérarchie fixe entre les droits et libertés mais une appréciation au cas par cas d'un équilibre entre les droits concernés à un instant donné et pour une situation particulière. L'équilibre entre les droits est donc fluctuant et dépend des choix faits par le législateur en fonction des circonstances. L'appréciation par les autorités publiques de cette conjoncture reste largement discrétionnaire et, à ce titre, le caractère temporaire d'une situation a pu justifier un déséquilibre en faveur d'un droit plutôt qu'un autre ${ }^{62}$.

47. CC, $\mathrm{n}^{\circ} 37$ de 2021; dans le même sens: CC, $\mathrm{n}^{\circ} 169$ de $2017, \mathrm{n}^{\circ} 338$ de $2003, \mathrm{n}^{\circ} 282$ de 2002 .

48. CC, $\mathrm{n}^{\circ} 37$ de 2021 .

49. Ibid.

50. Ibid.

51. CS, avis $\mathrm{n}^{\circ} 735$ de 2020 .

52. Loi 833 de 1978, art. 32 et décret législatif $n^{\circ} 112$ de 1998, art. 117; les ordonnances des présidents de région permettant de déroger aux lois régionales (décret législatif $\mathrm{n}^{\mathrm{O}} 1$ du 2 janvier 2018, art. 25).

53. Dont la validité est généralement d'un mois.

54. Décret-loi $\mathrm{n}^{\circ} 19$ du 25 mars 2020, art. 2.

55. CS, $\mathrm{n}^{\circ} 1553$ de 2020 ; D. Vese, «La strategia italiana per gestire l'emergenza COVID-19 e la sfida della condivisione dei poteri amministrativi», Persona e Amministrazione, $\mathrm{n}^{\circ}$ 2, 2020, p. 647, revue en ligne.

56. $\mathrm{CS}, \mathrm{n}^{\circ} 1553$ de 2020 .

57. CC, $\mathrm{n}^{\circ} 1146$ de 1988; P. Faraguna, Ai confini della Costituzione. Principi supremi e identità costituzionale, Milan, Franco Angeli, 2015.

58. Ce qui implique «la nécessaire conciliation entre le droit à la santé de chacun et le droit coexistant et réciproque des autres et l'intérêt de la collectivité» (CC, $\mathrm{n}^{\circ} 5$ de 2018). Les mesures prises au nom de la santé collective peuvent aussi avoir des effets sur la santé individuelle (L. Marilotti, «Contenimento del contagio, limitazioni domiciliari e salute psicofisica nell'attività di polizia sanitaria anti-coronavirus », Federalismi.it, n ${ }^{\circ} 1$, 2021, p. 235, revue en ligne).

59. CC, $\mathrm{n}^{\circ} 85$ de 2013 .

60. Ibid.; M. D’Amico, «Emergenza, diritti, discriminazioni», Associazione Gruppo di Pisa, juin 2020, p. 27.

61. A. Algostino, «Covid-19: primo tracciato per una riflessione nel nome della Costituzione», Osservatorio AIC, $\mathrm{n}^{\circ} 3,2020, \mathrm{p} .117$, revue en ligne. 62. Ibid. 
C'est ainsi que le juge constitutionnel a pu favoriser une restriction de certains droits dans des circonstances telles que la lutte contre le terrorisme ou la mafia ${ }^{63}$. Le droit à la santé n'échappe pas à ce cadre de référence et il a luimême fait l'objet de conciliation en sa défaveur ${ }^{64}$. On ne peut donc pas dire que ce droit s'impose intrinsèquement aux autres droits constitutionnellement protégés. Mais à l'inverse rien n'empêche qu'il soit prééminent dans certaines circonstances ${ }^{65}$. Or, la pandémie de Covid-19 forme une circonstance particulière évidente qui justifie le choix d'un équilibre entre les droits et libertés dans lequel le droit à la santé est prédominant. Dans ce cadre, le droit à la santé s'est manifesté essentiellement par des mesures de précaution, ce qui a conduit certains auteurs à soutenir que l'équilibre entre les droits est fondé sur la prépondérance du principe de précaution ${ }^{66}$.

Pour autant, la conciliation opérée au profit d'un droit plutôt qu'un autre a des limites. En effet, l'appréciation du législateur est bornée par la nécessité de préserver le «noyau essentiel» des droits affectés ${ }^{67}$. Toutefois, ce «noyau essentiel» ne renvoie pas à une définition précise et immuable mais forme un guide permettant l'évaluation de la conciliation entre les droits. De sorte que le noyau essentiel ne représente pas une concrétisation minimale et prédéterminée du droit affecté par la conciliation, mais sera respecté si la contrainte supportée par le droit considéré est raisonnable par rapport à la situation qui a justifié de faire prévaloir un autre droit ${ }^{68}$. Dans le cas de la pandémie, il faut déterminer si les restrictions apportées aux droits et libertés portent atteinte à ce noyau essentiel, et donc si elles sont raisonnables dans cette situation, au sens où elles sont dans un rapport de proportionnalité avec celle-ci ${ }^{69}$. Or, les fortes contraintes imposées par exemple à la liberté de circulation ont réduit quasiment à néant cette dernière ${ }^{70}$. Son noyau essentiel ne sera pourtant pas forcément remis en cause si on met ces restrictions extrêmes en rapport avec la situation extrême de la propagation de la pandémie (encore faut-il que l'intensité des deux éléments - contrainte et crise - reste conjointe, ce qui renvoie au nécessaire caractère temporaire de la restriction). Néanmoins, le juge constitutionnel n'a pas encore pu jouer son rôle de «boussole " ${ }^{71}$ et appliquer cette grille d'analyse aux décrets-lois adoptés pour contrer la pandémie ${ }^{72}$. C’est donc en raison d'une suprématie conjoncturelle que le droit à la santé justifie des contraintes sur les autres droits et libertés, lesquelles prennent la forme de mesures de précaution articulées par un exécutif national qui procède de fait à une conciliation entre droits à la place du législateur comme si, dans l'hypothèse de la pandémie, la nature scientifique de la situation créait un effet d'objectivation des priorités tendant à une sorte de consensus logique qui valide à son tour l'inutilité de l'intervention du législateur et la seule nécessité de l'efficacité incarnée par l'exécutif. On a pu dire à ce propos que les droits fondamentaux étaient «scientifiquement conditionnés par la Covid» ${ }^{73}$. Cette fragilité juridique des restrictions aux droits et libertés s'accompagne d'un contrôle limité de ces restrictions.

\section{Le contrôle limité des restrictions aux droits et libertés}

Les restrictions aux droits et libertés font l'objet d'un examen qui reste limité : le juge confronté à la crise montre une certaine frilosité (A) et la confrontation du droit à l'éducation avec le droit à la santé illustre bien les approximations du contrôle opéré (B).

\section{A. Une certaine frilosité du juge}

L'analyse des décisions de justice concernant le contrôle des mesures de restrictions des libertés fait apparaître que le juge a, de manière générale, validé les actes adoptés par les autorités nationales, alors qu'il a exercé un contrôle restreint sur les actes des autorités territoriales. Ainsi, la première question qui se pose est celle de la constitutionnalité des décrets-lois adoptés par le gouvernement, car ils représentent l'essentiel de la source des restrictions des droits et libertés. Or, le recours aux décrets-lois de l'article 77 de la Constitution permet au gouvernement de disposer d'une grande latitude, dans la mesure où il adopte un texte qui est ensuite converti en loi par le Parlement, la Cour constitutionnelle se bornant à vérifier que la condition de nécessité et d'urgence n'est pas manifestement absente ${ }^{74}$. La conversion par le

63. CC, $\mathrm{n}^{\circ} 15$ de 1982 .

64. Au nom de l'emploi (CC, $n^{\circ} 85$ de $2013, n^{\circ} 264$ de 2012).

65. CC, $\mathrm{n}^{\circ} 58$ de 2018

66. F. Scalia, «Principio di precauzione e ragionevole bilanciamento dei diritti nello stato di emergenza», Federalismi.it, $\mathrm{n}^{\circ}$ 32, 2020, p. $183-220$, revue en ligne.

67. Ibid., p. 208.

68. O. Chessa, «La misura minima essenziale dei diritti sociali: problemi e implicazioni di un difficile bilanciamento», Giurisprudenza Costituzionale, vol. 43, n 2, 1998, p. 1170-1187.

69. E. De Marco, «Situazioni di emergenza sanitaria e sospensioni di diritti costituzionali», Consulta online, n 2 , 2020, p. 375 , revue en ligne.

70. U. Fantigrossi, «Il diritto della pandemia : libertà di circolazione e trasporti nel governo dell'emergenza Covid-19», Sipotra, novembre 2020.

71. Pour reprendre l'expression de la présidente de la Cour constitutionnelle, M. Cartabia, dans son rapport annuel de 2020 (p. 26, en ligne: https:// www.cortecostituzionale.it/documenti/relazione_cartabia/1_relazione.pdf).

72. Alors même qu'il y a une «exigence d'interpellation de la Cour» (A. Lauro, «Urgenza e legalità ai tempi del Covid-19», Rivista di BioDiritto, $\mathrm{n}^{\circ} 1,2020$, p. 152, revue en ligne).

73. R. Dagostino, «Emergenza, pandemia, crisi del sapere e funzioni pubbliche necessitate. I diritti fondamentali scientificamente condizionati al tempo del Covid-19", Persona e Amministrazione, n 2, 2020, p. 403-423, revue en ligne.

74. A. Sperti, «La Corte costituzionale e il controllo sui vizi formali del decreto-legge», Osservatorio AIC, n 6, 2020, p. 376 , revue en ligne. 
législateur ne les immunise pas pour autant contre une éventuelle inconstitutionnalité 75 . Cependant, la plupart des décrets-lois n'ont pas été remis en cause directement devant la Cour constitutionnelle (du fait du consensus initial, mais aussi de la courte validité de certains d'entre eux...). D'ailleurs, celle-ci a admis implicitement que le recours aux décrets-lois et aux DPCM est conforme à la Constitution $^{76}$, même si elle renvoie au juge administratif la compétence de contrôler les DPCM, et que «le modèle proposé par la législation en vigueur [...] paraît conforme à la conception constitutionnelle, même s'il n'est pas l'unique moyen de mettre en œuvre celle-ci »77. Dans le même sens, la suspension de la prescription en matière pénale pour des actes commis avant le 9 mars 2020 établie par le décret-loi $\mathrm{n}^{\circ} 18$ du 17 mars 2020 a été justifiée par le but de protéger le bien de la santé collective pour contenir le risque de contagion dans un moment exceptionnel d'urgence sanitaire car elle était raisonnable et proportionnelle ${ }^{78}$.

Mais l'essentiel des restrictions a été adopté par les DPCM, qui ne sont vérifiés ni par la Cour constitutionnelle (car il s'agit d'actes administratifs ${ }^{79}$ ), ni par le législateur ou le président de la République (contrairement aux décretslois) et qui ne peuvent donc être contrôlés que par le juge administratif. Or, pour des raisons identiques à celles avancées pour les décrets-lois (consensus, sidération, succession rapide des mesures...), les DPCM n'ont fait l'objet que de quelques recours directs. Certes, le Conseil d'État, en renvoyant l'affaire au tribunal administratif régional (TAR) du Latium ${ }^{80}$, a estimé que le recours contre les DPCM du 3 novembre et du 3 décembre 2020 nécessitera d'examiner "une éventuelle inconstitutionnalité ${ }^{81}$. En effet, les DPCM ne peuvent pas être directement contrôlés par le juge constitutionnel, mais ce dernier peut être saisi par un juge du fond qui soulève l'inconstitutionnalité des décrets-lois fondant les DPCM. Pour le reste, concernant le DPCM du 3 novembre 2020, le TAR du Latium admet que

[...] la décision de l'autorité de réduire pour une période de temps limitée un intérêt certainement significatif pour le bien-être individuel et de la collectivité, tel que la fréquentation des musées et des autres lieux de culture, n'est pas manifestement déraisonnable au regard de la gravité particulière de l'urgence sanitaire en cours $^{82}$.

Et le Conseil d'État confirme le refus du TAR: le «défaut de proportionnalité» n'est pas présent car

[...] dans les limites du contrôle qui relève du juge administratif, d'autant plus en présence de mesures comme celle examinées qui sont l'expression d'un haut degré d'appréciation discrétionnaire parce qu'elles sont le fruit d'une comparaison très délicate entre des intérêts publics qui sont tous importants, la décision de l'autorité de limiter temporairement la fréquentation des musées et des autres lieux culturels n'est pas manifestement déraisonnable ${ }^{8_{3}}$.

Ainsi, lors des rares recours examinés, le juge administratif s'est appuyé sur un examen de la proportionnalité de la mesure en cause mais en ne procédant pas systématiquement à un "triple test» de nécessité, du caractère adapté et de la proportion proprement dite. Une autre voie de droit a aussi été empruntée sans plus de succès: un recours extraordinaire au président de la République ${ }^{84}$ a été introduit pour demander l'annulation des DPCM adoptés entre mars et mai; le Conseil d'État a rendu un avis (qui doit être obligatoirement suivi) au président de la République par lequel il propose de rejeter cette demande pour irrecevabilité, les actes en question ne produisant plus d'effet à la date d'introduction du recours ${ }^{85}$. Aussi, on peut dire que le contrôle des actes nationaux a globalement validé les mesures adoptées par le pouvoir central. Le bilan est plus contrasté concernant les actes régionaux et locaux, sans toutefois se détacher de la ligne générale de retenue dont fait preuve le juge.

En effet, les ordonnances des présidents de région et des maires ont fait l'objet d'un contentieux devant les TAR et le Conseil d'État ${ }^{86}$ : alors que, normalement, les décisions de suspension en urgence et à juge unique devant les TAR ne font l'objet que d'un recours exceptionnel en appel devant le Conseil d'État ${ }^{87}$, le décret-loi $n^{\circ} 18$ de 2020 a étendu ce recours pour améliorer la garantie

75. CC, $\mathrm{n}^{\circ} 94$ de $2016, \mathrm{n}^{\circ} 154$ de $2015, \mathrm{n}^{\circ} 32$ de $2014, \mathrm{n}^{\circ} 220$ de 2013.

76. CC, $\mathrm{n}^{\circ} 37$ de 2021 .

77. Ibid.

78. CC, $\mathrm{n}^{\circ} 278$ de 2020 ; G. L. Gatta, «Emergenza Covid e sospensione della prescrizione del reato", Sistema penale, 26 décembre 2020 , revue en ligne; de même, le décret-loi $\mathrm{n}^{\circ} 29$ du 10 mai 2020 permettant à un juge de remplacer la détention par l'assignation à résidence pour des raisons liées à l'urgence sanitaire est conforme au droit à la santé (CC, nº 245 de 2020).

79. R. Cavallo Perin, "Pandemia 2020: decreti e ordinanze d'emergenza», Giustizia Insieme, mai 2020, revue en ligne.

80. Après un rejet devant le TAR du Latium ( $\mathrm{n}^{\circ} 7727$ de 2020), annulé par le Conseil d'État ( $\mathrm{CS}, \mathrm{n}^{\circ} 115$ de 2021$)$, ce dernier a renvoyé l'affaire au TAR pour une audience fixée au 14 juillet 2021 (recours $n^{\circ} 9159$ de 2020).

81. CS, $\mathrm{n}^{\circ} 115$ de 2021.

82. TAR Latium, $\mathrm{n}^{\circ} 7571$ de 2020.

83. CS, $\mathrm{n}^{\circ} 7255$ de 2020 .

84. Le président de la République doit répondre au recours contre un acte administratif définitif sur la base de la proposition faite par le gouvernement et ce dernier ne peut s'écarter de la position du Conseil d'État que par une délibération du Conseil des ministres (article 8 du décret du président de la République no 1119 du 24 novembre 1971; voir «Ricorso straordinario », Enciclopedia Treccani, en ligne: https://www.treccani.it/ enciclopedia/ricorso-straordinario).

85. CS, avis $\mathrm{n}^{\circ} 422$ de 2021 : le recours a été introduit le 13 juillet 2020, alors que les DPCM avaient une validité limitée, le dernier de la liste des actes attaqués prenant fin le 14 juin 2020.

86. La Cour constitutionnelle refuse de les contrôler au motif qu’elles n’ont pas force de loi (CC, nº 8 de 1956, nº 4 de 1977); M. Midiri, « Emergenza, diritti fondamentali, bisogno di tutela : le decisioni cautelari del giudice amministrativo ", Dirittifondamentali.it, $\mathrm{n}^{\circ}$ 2, 2020, p. 61-71, revue en ligne.

87. CS, n 5650 de $2014, n^{\circ} 5971$ de 2018. 
des requérants ${ }^{88}$. Les juges régionaux ont été saisis de très nombreux recours, d'abord concernant plutôt des ordonnances prises par des maires puis, dans un second temps, essentiellement des ordonnances adoptées par des présidents de région ${ }^{89}$. L'ordonnance du TAR de Toscane du 5 mars $2021^{9 \circ}$ est exemplaire sur le plan de la méthode de contrôle que le juge doit normalement suivre. Le contentieux portait sur l'ordonnance du président de la région de Toscane qui interdisait le retour dans la région des personnes qui y ont leur domicile s'ils n'ont pas un médecin généraliste dans cette même région ${ }^{91}$. Pour qu'une telle ordonnance soit légale, alors qu'elle réglemente une liberté fondamentale comme la libre circulation, le TAR rappelle qu'elle: 1) doit s'appuyer sur une « extraordinaire nécessité et urgence»; 2) doit adopter des mesures adaptées et proportionnelles au risque épidémiologique sur le territoire régional;3) ne peut s'appliquer que pour un temps limité (jusqu'à l'intervention du prochain DPCM) ; 4) doit se fonder sur l'aggravation de la situation épidémique dans la région. À cela s'ajoute que seule une restriction supplémentaire et non un assouplissement des mesures nationales peut être décidée par cette voie ${ }^{92}$. En l'occurrence, sans aggravation de la situation et s'écartant de la législation nationale, l'ordonnance a été suspendue. Sur le premier point (nécessité), le juge est souvent facile à convaincre, dès lors que le principe de précaution est mis en avant (et ce dernier est souvent reconnu comme une évidence). Mais il s'attarde parfois à vérifier cette nécessité, ce qui tend alors à recouper le quatrième point (la progression locale du virus plus forte ou l'exigence de la campagne de vaccination, l'absence d'obligation de porter les masques pour les plus jeunes enfants... ${ }^{93}$. Concernant le deuxième point, l'adaptation est absorbée par le contrôle de proportionnalité stricto sensu tandis que la vérification de cette dernière se borne à examiner l'absence de caractère déraisonnable de la mesure (en raison du pouvoir d'appréciation discrétionnaire de l'autorité publique, du délicat arbitrage entre droits constitutionnels également protégés, sachant que la protection de la santé est prééminente, et même du caractère temporaire qui joue en faveur du caractère proportionnel ${ }^{94}$ ). Cette ligne jurisprudentielle, bien que souvent un peu confuse, conduit globalement à valider plus facilement les restrictions quand l'épidémie progresse et que le droit à la santé est présenté comme une "priorité absolue ${ }^{95}$, transformant le principe de précaution en recherche du "risque zéro" même sans spécificité locale ${ }^{96}$. En examinant plus précisément le cas de la confrontation entre le droit à la santé et le droit à l'éducation, on prendra mieux la mesure du caractère approximatif du contrôle opéré.

\section{B. Droit à la santé versus droit à l'éducation, une illustration d'un contrôle approximatif}

\author{
L'article 34 de la Constitution précise que:
}

L'école est ouverte à tous. L'instruction de base, dispensée durant au moins huit ans, est obligatoire et gratuite. Les élèves doués et méritants, même s'ils sont dépourvus de moyens financiers, ont le droit d'atteindre les degrés les plus élevés des écoles. La République rend ce droit effectif par des bourses d'études, des allocations aux familles et par d'autres moyens devant être attribués par concours.

Le droit à l'éducation contribue ainsi à l'accès à la culture et à la formation des citoyens républicains proclamés aux articles $1^{\text {er }}$ et 9 de la Constitution ${ }^{97}$ et à l'épanouissement de la personne humaine ${ }^{98}$. Or, au titre des mesures de restriction pour lutter contre la pandémie, le libre accès à l'éducation a été pratiquement éliminé (fermeture des établissements, remplacement de l'enseignement présentiel par un enseignement à distance) avec des conséquences sur le creusement des inégalités ${ }^{99}$. Ainsi, le gouvernement a disposé que, dans toutes les zones ${ }^{100}$, l'enseignement de la maternelle à la sixième est assuré en présentiel et que, hors zone rouge, l'enseignement présentiel est assuré

88. M. Midiri, «Emergenza...», p. 65.

89. Voir entre autres: une interdiction faite aux employés de se rendre sur leur lieu de travail (TAR Calabre, $\mathrm{n}^{\circ} 165$ de 2020 et CS, $\mathrm{n}^{\circ} 1553$ de 2020 ); une interdiction de se déplacer à proximité de son habitation (TAR Sicile, $\mathrm{n}^{\circ} 458$ de 2020); une limitation du nombre de sorties pour faire ses emplettes (TAR Sardaigne, $\mathrm{n}^{\circ} 122$ de 2020).

90. TAR Toscane, $\mathrm{n}^{\circ} 334$ de 2021.

91. Ordonnance $\mathrm{n}^{\circ} 3$ du 22 janvier 2021.

92. TAR Lombardie, $\mathrm{n}^{\circ} 634$ de 2020.

93. TAR Campanie no 302 de $2021 ;$ CS, no 749 de 2021.

94. Ibid.

95. Voir entre autres: CS, $\mathrm{n}^{\circ} 749$ du 15 février 2021.

96. TAR Sicile, $n^{\circ} 458$ de 2020

97. M. Benvenuti, «Articolo 34 ", in La Costituzione italiana. Commento articolo per articolo, F. Clementi, L. Cuocolo, F. Rosa, G. E. Vigevani (dir.), Bologne, Il Mulino, 2018, p. 226.

98. Constitution italienne, art. 3: «La République a le devoir d'éliminer les obstacles d'ordre économique et social qui, en limitant la liberté et l'égalité des citoyens, empêchent l'épanouissement de la personne humaine»; art. 2: «La République reconnaît et garantit les droits inviolables de l'homme, comme individu et comme membre de formations sociales où se développe sa personnalité [...]"; voir CC, nº 215 de 1987 et J. Fougerouse, "Bonheur, épanouissement de la personne et bien-être en droit constitutionnel italien », in Bonheur et bien-être dans le droit des États, J. Fougerouse, F. Lemaire (dir.), Paris, Mare \& Martin, 2021, à paraître.

99. A. Nuzzaci, R. Minello, N. Di Genova, S. Madia, «Povertà educativa in contesto italiano tra istruzione e disuguaglianze. Quali gli effetti della pandemia ?", La rivista internazionale di EdaForum, vol. 17, $\mathrm{n}^{\circ}$ 36, 2020, p. 76-92; G. Chinaglia, "Covid-19 e misure di contenimento del contagio: l'impatto sugli studenti», in Tra emergenza, eccezione e precauzione, L. Imarisio, M. Malvicini, G. Sobrino (dir.), Turin, Università di Torino, 2020 , p. 187.

100. Les zones rouges, oranges et jaunes créées par le décret-loi nº 33 du 16 mai 2020 correspondent à un groupe de restrictions. 
jusqu'à la quatrième ${ }^{101}$. Les autorités régionales peuvent intervenir pour imposer des restrictions supplémentaires si elles sont temporaires, motivées, nécessaires et qu'il n'y a pas d'autres moyens moins restrictifs ${ }^{102}$.

Les présidents de région ayant adopté de nombreuses ordonnances d'urgence, le juge administratif a été saisi de recours en suspension et en annulation qui lui ont donné l'occasion de contrôler la conciliation entre le droit à la santé et le droit à l'éducation ${ }^{103}$. Il est vrai que cette confrontation est un combat de géants :

[...] le droit à la santé - seul à être aussi un intérêt collectif se trouve au sommet des valeurs constitutionnelles, en ce qu'il permet de jouir des libertés et des droits protégés par la Constitution, mais le droit à l'éducation vient tout juste derrière en ce qu'il permet l'accès au travail sur lequel la République est fondée et parce qu'il est un moyen de permettre [...] l'épanouissement de la personne humaine ${ }^{104}$.

Aussi, il n'est pas surprenant que le Conseil d'État affirme, à propos de la suspension de toutes les activités d'enseignement en présentiel décidée par le président de la région Campanie, que

[...] les mesures de ce genre, en raison de la conciliation nécessaire entre les droits - à la santé et à l'instruction ayant un rang et une protection constitutionnels, doivent se fonder sur des évaluations scientifiques précises, spécifiques et mises à jour, permettant de faire apparaître des données cohérentes avec l'ampleur des restrictions ${ }^{105}$.

Mais, une fois ce schéma de contrôle tracé, le juge estime qu'il ne lui appartient pas de vérifier les éléments qui servent de base à la décision mais « seulement d'évaluer si le choix administratif n'est pas [...] déraisonnable $»^{106}$. Cette retenue du juge, et cette seule appréciation du caractère déraisonnable ${ }^{107}$, s'écartant du triple test de proportionnalité a été critiquée ${ }^{108}$ et il est vrai que les décisions des juges ne sont pas toujours rigoureuses sur ce point ${ }^{109}$.

L'examen du caractère raisonnable de la mesure (reconnu le plus souvent ${ }^{110}$ ) concentre l'essentiel du contrôle car la vérification de la nécessité et du caractère adapté est généralement laissée de côté ${ }^{111}$, comme si les mesures allaient d'elles-mêmes dès lors qu'elles sont fondées sur la défense de la santé ${ }^{112}$. Dans certains cas, le juge semble faire la démarche de pratiquer ces étapes du contrôle:

[...] dans l'hypothèse d'un conflit entre les droits en question, l'issue n'est pas nécessairement et automatiquement la mise à l'écart de l'éducation au profit de la santé, mais en premier lieu la recherche des mesures permettant leur conciliation et, seulement dans les cas où il est impossible d'y parvenir, le droit à l'éducation (en présentiel) est écarté ${ }^{113}$.

Mais, très vite, la retenue l'emporte, alors que, si l'urgence permet d'écarter l'application des règles normales au profit d'une simple compétence conférée à une autorité à qui est confié un objectif, c'est sous la réserve qu'il n'y ait pas d'autres moyens pour faire face à l'urgence et que les mesures prises sont provisoires et proportionnées ${ }^{114}$. Le contrôle de proportionnalité, au sens du caractère raisonnable de la mesure, n'est pas même déployé complètement: le juge met en balance le principe de précaution sanitaire avec l'atteinte au droit à l'éducation ${ }^{115}$, alors que cette conciliation est biaisée dès le départ. Ainsi, le premier est entendu extensivement et le second restrictivement, ce qui conduit de manière quasi inéluctable à un résultat presque constamment favorable à la santé publique. Le droit à la santé, sous l'angle de la précaution, englobe ainsi la nécessité d'éviter une défaillance des services de santé en raison du nombre de malades, quand bien même le lien avec l'ouverture des écoles n'est pas forcément établi et alors que l'administration dispose d'une grande latitude pour apprécier cette précaution. À l'inverse, l'atteinte au droit à l'éducation est minimisée:

[...] l'altération des autres droits impliqués n'est en fait pas absolue, en raison de la garantie des activités scolaires grâce à l'enseignement numérique à distance et en l'absence de démonstration de l'impossibilité de concilier l'activité professionnelle des parents et l'assistance familiale aux enfants mineurs ${ }^{116}$.

101. DPCM du 3 novembre 2020.

102. Décret-loi $\mathrm{n}^{\circ} 33$ de 2020 , art. $1^{\text {er }}$, al. 16 .

103. L. Dell'Atti, «Bilanciare istruzione e salute. Considerazioni brevi su strumenti unitari e leale collaborazione a partire da talune ordinanze regionali in materia di sospensione della didattica "in presenza" ", Osservatorio AIC, $\mathrm{n}^{\circ}$ 1, 2021, p. 87-107, revue en ligne.

104. TAR Calabre, n' 2075 de 2020.

105. CS, $\mathrm{n}^{\circ} 1034$ de 2021 .

106. Ibid.

107. Sur laquelle des critiques se sont élevées: V. Baldini, «Proporzionalità e adeguatezza versus prevenzione nel controllo di razionalità delle misure di contrasto all'emergenza sanitaria», Dirittifondamentali.it, $\mathrm{n}^{\circ}$ 1, 2021, p. 136-152, revue en ligne.

108. I. Romano, «Spunti di riflessione sull'applicazione del principio di proporzionalità ai tempi del Covid-19", Persona e Amministrazione, $\mathrm{n}^{\circ}$ 2, 2020, p. 519, revue en ligne.

109. TAR Calabre, $n^{\circ} 2075$ de 2020.

110. M. Midiri, «Emergenza...», p. 67.

111. On a relevé un seul cas où le juge a demandé au président de région de réexaminer son ordonnance imposant les cours à distance pour «prendre en compte les moyens dont disposent les structures pédagogiques" (TAR Basilicate, $\mathrm{n}^{\circ} 272$ de 2020).

112. Ainsi, le juge ne vérifie pas la possibilité de prendre des mesures restrictives alternatives à la fermeture des établissements scolaires.

113. TAR Calabre, $\mathrm{n}^{\circ} 2075$ de 2020 ; TAR Campanie, $\mathrm{n}^{\circ} 1921$ de 2020.

114. CS, $\mathrm{n}^{\circ} 868$ de 2010.

115. CS, $\mathrm{n}^{\circ} 1034$ de 2021

116. Ibid.; dans le même sens: «rien ne prouve le préjudice du mineur privé des services socio-éducatifs» $\left(\mathrm{CS}, \mathrm{n}^{\circ} 749\right.$ de 2021$)$. 
Si le juge reconnaît parfois les problèmes soulevés par l'enseignement à distance, il n'en tire pas de conséquences ${ }^{117}$. Dans ces conditions, la plupart des ordonnances de présidents de région ont été validées (à part quelques cas, tel ce TAR qui relève qu' «en choisissant le risque zéro » la région a adopté une mesure «non proportionnelle ${ }^{118}$ ). De même, le caractère temporaire de ces ordonnances régionales joue en faveur de leur légalité ${ }^{119}$, alors qu'elles s'inscrivent de fait dans la durée (depuis mars 2020 pour une part des élèves) ${ }^{120}$.

Enfin, deux affaires récentes montrent comment le juge adapte son contrôle à l'évolution de la pandémie. Dans la première, alors qu'une ordonnance du président de la région des Abruzzes prorogeait l'enseignement à distance pour tous les niveaux scolaires, le juge estime qu'il ne peut apprécier l'évaluation faite par le président de région concernant les différents territoires de la région où l'enseignement en présentiel est suspendu ${ }^{121}$, que s'il est

[...] manifestement déraisonnable et incongru par rapport aux données scientifiques sur lequel il s'appuie car il s'agit de comparer le droit à l'éducation (et les restrictions dérivant de l'enseignement à distance) avec le droit à la santé de l'ensemble de la population en période de pandémie ${ }^{122}$.

Dans la seconde affaire, le juge, saisi d'une ordonnance du président de la région Ombrie qui interdisait l'enseignement en présentiel dans les lycées de Pérouse, précise qu'il est "parfaitement conscient de la grave restriction du droit des lycéens à un apprentissage complet et à la jouissance de l'offre d'enseignement par un contact direct et personnel avec les enseignants et les autres lycéens $»^{123}$ mais que la propagation du virus ayant «nettement augmenté y compris en Ombrie ${ }^{124}$ l'arrêt de l'enseignement en présentiel s'est imposé «pour donner la priorité à la protection de la santé des citoyens, valeur prééminente pendant une pandémie ${ }^{125}$. Aussi, dans ces circonstances, toute possibilité d'évaluer sélectivement les zones soumises à un risque plus ou moins grand (évaluation qui relève de la responsabilité des autorités nationales et locales qui en ont la charge) est exclue ${ }^{126}$, de même que toute possibilité de donner la priorité «dans le cadre de la nécessaire, difficile et par certains aspects douloureuse, pondération entre les valeurs constitutionnellement protégées $»^{127} \mathrm{au}$ droit à l'enseignement en présentiel «sur la plus absolue précaution eu égard au danger de propagation de la contagion avec les effets dévastateurs sur la santé publique et le fonctionnement des hôpitaux ${ }^{128}$. De ces deux positions on peut déduire un certain flottement du juge sur la nature et l'intensité du contrôle qu'il opère. Dans la seconde affaire, le juge se penche vaguement sur la nécessité de la mesure (accroissement local de la propagation du virus, mais sans s'attarder sur les données scientifiques présentées par le requérant) tandis que cette nécessité est seulement implicite dans la première affaire ${ }^{129}$. Quant au caractère adapté, il est seulement évoqué (congruité) concernant l'affaire des Abruzzes, tandis que le contrôle du caractère manifestement déraisonnable prend la place de celui de la proportionnalité. Or dans l'affaire de l'Ombrie ce dernier semble balayé, sous prétexte que le juge ne veut pas donner la priorité à l'enseignement sur la santé, alors que le contrôle du caractère manifestement déraisonnable suppose un examen minimal du choix fait par l'autorité: on peut se demander si le juge ne cède pas à la panique en parlant de «la plus absolue précaution ${ }^{130}$. Au travers de ces quelques exemples, on voit donc bien apparaître le caractère approximatif du contrôle qui cache mal la retenue et le pragmatisme du juge ${ }^{131}$, lequel renvoie souvent les autorités à leurs "responsabilités $»^{132}$.

Finalement, l'analyse des décisions juridictionnelles montre que, dans un contexte exceptionnel, les droits et libertés peuvent être largement restreints, sans que des obstacles institutionnels et constitutionnels s'y opposent réellement et sans que les juges résistent fortement à la tempête d'une nécessité présentée comme une solution unique.

117. «La suppression [...] d’un enseignement en présentiel est préjudiciable sur le plan de la formation au niveau psychologique, éducatif et de la socialisation des enfants» (TAR Calabre, $n^{\circ} 2075$ de 2020); dans le même sens voir CS, $n^{\circ} 1034$ de 2021; S. Prisco, «La didattica universitaria a distanza: "filosofia", opportunità, limiti e rischi», Dirittifondamentali.it, n 2, 2020, p. 1083, revue en ligne; B. Bruschi, P. Ricchiardi, «Effetti della chiusura delle scuole sull'apprendimento degli studenti ", Il Piemonte delle Autonomie, n 2, 2020.

118. TAR Calabre, $\mathrm{n}^{\circ} 2075$ de 2020

119. TAR Campanie, $n^{\circ} 1921$ de 2020.

120. Sur l'installation de l'urgence dans le temps, voir U. Ronga, «Il governo nell'emergenza (permanente). Sistema delle fonti e modello legislativo a partire dal caso Covid-19", Nomos, $\mathrm{n}^{\circ} 1,2020$, revue en ligne.

121. CS, $\mathrm{n}^{\circ} 1034$ de 2021.

122. CS, no 1448 de 2021 .

123. CS, $\mathrm{n}^{\circ} 1234$ de 2021.

124. Ibid.

125. Ibid.

126. Le Conseil d'État contredit l'orientation suivie par le TAR de Calabre ( $n^{\circ} 609$ de 2020) qui avait suspendu la fermeture décidée eu égard à sa généralité au regard des particularités locales.

127. CS, no 1234 de 2021.

128. Ibid.

129. La nécessité est parfois mieux évaluée (TAR Émilie-Romagne, $\mathrm{n}^{\circ} 120$ de 2021; TAR Campanie, $\mathrm{n}^{\circ} 302$ de 2021).

130. Voir aussi CS, $\mathrm{n}^{\circ} 884$ de 2021.

131. Certains parlent «d'indulgence» (C. Amodio, I. Boucobza, «Quand le président du Conseil limite les libertés au nom du droit à la santé publique... Imbroglio sur la constitutionnalité de l'état d'urgence sanitaire en Italie», La revue des droits de l'homme, juillet 2020, note 82, en ligne: https:// journals.openedition.org/revdh/10413), d'autres vont jusqu'à dénoncer une privation de protection judiciaire (G. Pernice, «Le ordinanze di necessità e di urgenza alla luce del principio di proporzionalità », Persona e Amministrazione, nº 2, 2020, p. 453, revue en ligne).

132. CS, $\mathrm{n}^{\circ} 1034$ de $2021, \mathrm{n}^{\circ} 1234$ de 2021 . Ce renvoi fait référence à la formule de l'article 77 de la Constitution relatif aux décrets-lois. 